\title{
Recent results from MAGIC
}

\author{
Alessandro De Angelis* ${ }^{* \dagger}$ \\ INFN and INAF Trieste - University of Udine and LIP/IST Lisboa \\ E-mail: alessandro.de.angelis@cern.ch
}

\begin{abstract}
Ground-based gamma-ray astronomy is part of a new field of fundamental research of Astroparticle Physics, that recently made spectacular discoveries. Nearly all important discoveries have been made by Imaging Air Cherenkov Telescopes (IACT) which allow one to detect Cherenkov light from extended air showers initiated by cosmic gamma-rays and charged cosmic particles. The MAGIC telescope is a new generation IACT located at La Palma, Canary Islands, Spain. Composed by two antennas with $17 \mathrm{~m}$ diameter each, MAGIC is equipped with the largest optical reflectors in the world, and it has the lowest threshold energy ( $25 \mathrm{GeV})$. MAGIC started operations in 2004 in the single-detector configuration, and in 2009 as a stereo detector. Since then, it has discovered many new sources and classes of sources, both galactic and extragalactic. Here some highlights from the most recent results are presented.
\end{abstract}

The Extreme sky: Sampling the Universe above $10 \mathrm{keV}$

October 13-17 2009

Otranto (Lecce) Italy

\footnotetext{
*Speaker.

$\dagger$ On behalf of the MAGIC Collaboration.
} 


\section{Introduction}

The Major Atmospheric Gamma Imaging Cherenkov (MAGIC) telescope is a last generation instrument for very high energy (VHE, conventionally above $30 \mathrm{GeV}$ [1]) $\gamma$-ray observations exploiting the Imaging Air Cherenkov technique. This kind of instrument images the Cherenkov light produced in the cascade initiated by a particle hitting the atmosphere [1].

MAGIC (Figure 1) is composed by two $17 \mathrm{~m}$ diameter telescopes located on the Roque de los Muchachos Observatory, in La Palma (Spain), and it is thus the largest optical reflector in the world. MAGIC incorporates a number of technological improvements in its design and achieves the lowest energy threshold $(55 \mathrm{GeV}$ with the standard trigger, $25 \mathrm{GeV}$ with a special trigger, activated in particular conditions) among the instruments of its kind. The signal digitization utilizes 2GSample/s Flash ADCs.

The sensitivity for a single telescope is such that, at a flux peak energy of $300 \mathrm{GeV}$, a source as faint as of $1.6 \%$ of the Crab Nebula can be detected at $5 \sigma$ in 50 hours of observations. The relative energy resolution above $200 \mathrm{GeV}$ is below $25 \%$; the angular resolution is about 0.1 degrees. MAGIC is also unique among Cherenkov telescopes by its capability to operate under moderate illumination (i.e., moon or twilight); this allows to increase the duty cycle.

Operating as a single telescope since 2004, MAGIC has started stereo observations at the end of 2009, with the basic parameters summarized in the following Table (in comparison with the other large gamma-ray telescopes, H.E.S.S. in Namibia and VERITAS in Arizona).

\begin{tabular}{lccccccc}
\hline Instrument & \# Tels. & $\begin{array}{c}\text { Tel. Area } \\
\left(\mathrm{m}^{2}\right)\end{array}$ & $\begin{array}{c}\text { Field of View } \\
\left({ }^{\circ}\right)\end{array}$ & $\begin{array}{c}\text { Total A } \\
\left(\mathrm{m}^{2}\right)\end{array}$ & $\begin{array}{c}\text { Thresh. } \\
(\mathrm{TeV})\end{array}$ & $\begin{array}{c}\text { Ang. res. } \\
\left({ }^{\circ}\right)\end{array}$ & $\begin{array}{c}\text { Sensitivity } \\
(\% \text { Crab })\end{array}$ \\
\hline H.E.S.S. & 4 & 107 & 5 & 428 & 0.1 & 0.06 & 0.7 \\
MAGIC & 2 & 236 & 3.5 & 472 & 0.05 & 0.08 & 0.8 \\
VERITAS & 4 & 106 & 4 & 424 & 0.1 & 0.07 & 0.7 \\
\hline
\end{tabular}

MAGIC has developed a physics program which includes topics of both fundamental physics and astrophysics. In this paper we highlight some of the MAGIC latest contributions to Galactic and extragalactic astrophysics, with emphasis on fundamental physics.

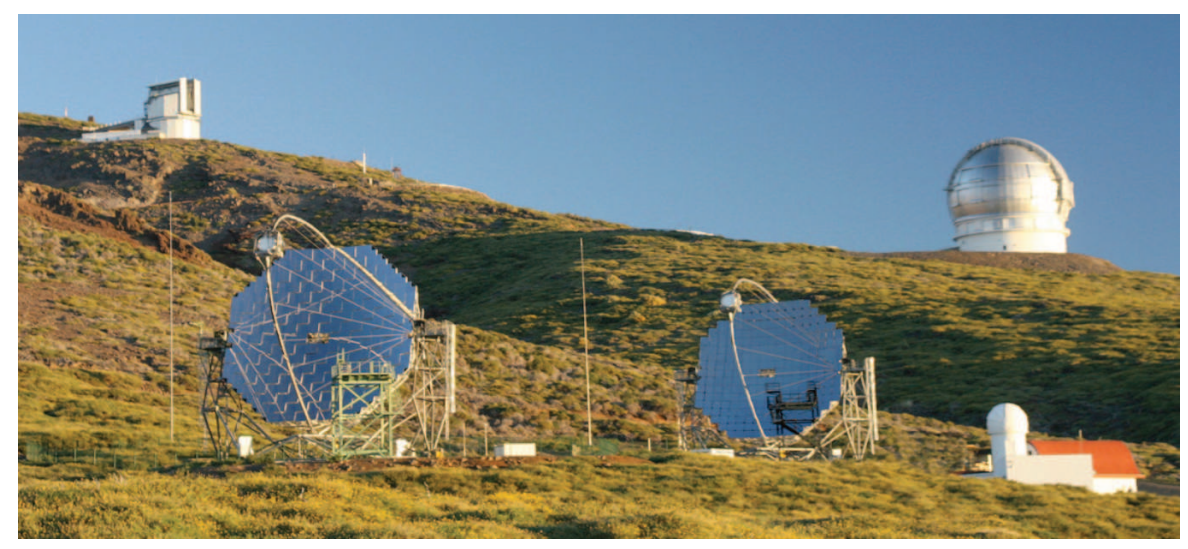

Figure 1: The MAGIC telescopes. 


\section{Highlights of Galactic observations}

In this section the latest MAGIC results on Galactic astrophysics are reviewed. MAGIC has discovered 4 new Galactic sources at VHE: MAGIC J0616+225 [2], positionally consistent with the supernova remnant IC 443; the first binary source for which periodical variability at VHE has been evidenced, LSI +61 303 [3]; Cygnus X-1 [4]; the pulsar from Crab [5]. They are all likely to indicate new classes of emitters. For some 20 other Galactic sources MAGIC has confirmed the discovery, performing flux and morphology measurements. Finally, MAGIC has published significant flux upper limits for interesting classes of sources, like, e.g, Wolf-Rayet binaries [6].

\subsection{Shell-type Supernova Remnants: Cassiopeia A and MAGIC J0616+225}

MAGIC observed the shell-type supernova remnant (SNR) Cassiopeia A during 47 goodquality hours, and detected a point-like source of VHE $\gamma$-rays above $\sim 250 \mathrm{GeV}$ [7]. The measured spectrum is consistent with a power law with a differential flux at $1 \mathrm{TeV}$ of $\left(1.0 \pm 0.1_{\text {stat }} \pm 0.3_{\text {sys }}\right) \times$ $10^{-12} \mathrm{TeV}^{-1} \mathrm{~cm}^{-1} \mathrm{~s}^{-1}$ and a spectral index of $\Gamma=2.4 \pm 0.2_{\text {stat }} \pm 0.2_{\text {sys }}$. The spectrum measured by MAGIC is consistent with that measured by HEGRA [8] 8 years before for the energies above $1 \mathrm{TeV}$, i.e., where the two measurements overlap. The MAGIC results favor a hadronic scenario for the $\gamma$-ray production, since a leptonic origin of the $\mathrm{TeV}$ emission would require low magnetic field intensities, which are difficult to reconcile with the high values required to explain the rest of the broad-band spectrum.

In addition, MAGIC has discovered a new source of VHE $\gamma$-rays located close to the Galactic plane, which has been named MAGIC J0616+225 [2]; this source is spatially coincident with the SNR IC 443. The measured energy $(E)$ spectrum is well fitted by a power law with spectral index of $\Gamma=3.1 \pm 0.3$. MAGIC J0616+225 is point-like for the MAGIC spatial resolution, and appears to be displaced from the center of the SNR shell. The observed VHE radiation may be due to $\pi^{0}$ decays from the interaction between cosmic rays accelerated in IC 443 and the dense molecular cloud.

\subsection{Compact binaries: Cygnus X-1 and LS I +61 303}

Cygnus $\mathrm{X}-1$ is the best established candidate for a stellar mass black-hole and one of the brightest X-ray sources in the sky. MAGIC has observed it for 40 hours along 26 different nights between June and November 2006. The MAGIC observations obtained evidence (4.1 $\sigma$ post-trial significance) of a short-lived, intense flaring episode during 24th September 2006, in coincidence with a high flux observed in X-rays. The detected signal is point-like. The energy spectrum fits well extrapolations from Integral.

LS I +61 303 is a binary system containing a main-sequence star together with a compact object (neutron star or black hole), which displays periodic emission throughout the spectrum from radio to X-ray wavelengths. Observations with MAGIC have determined that this object produces $\gamma$-rays up to at least $\sim 4 \mathrm{TeV}$ [3], and that the emission is periodically modulated by the orbital motion $\left(P_{\mathrm{TeV}}=(26.8 \pm 0.2) \mathrm{d}\right)[9]$. The peak of the emission is found at orbital phases not coincident with the periastron. 
In a multiwavelenght campaign involving radio (VLBA, e-EVN, MERLIN), X-ray (Chandra) and $\mathrm{TeV}$ (MAGIC) observations [10], a hint of temporal correlation between the $\mathrm{X}$-ray and $\mathrm{TeV}$ emissions and evidence for radio/TeV non-correlation were found.

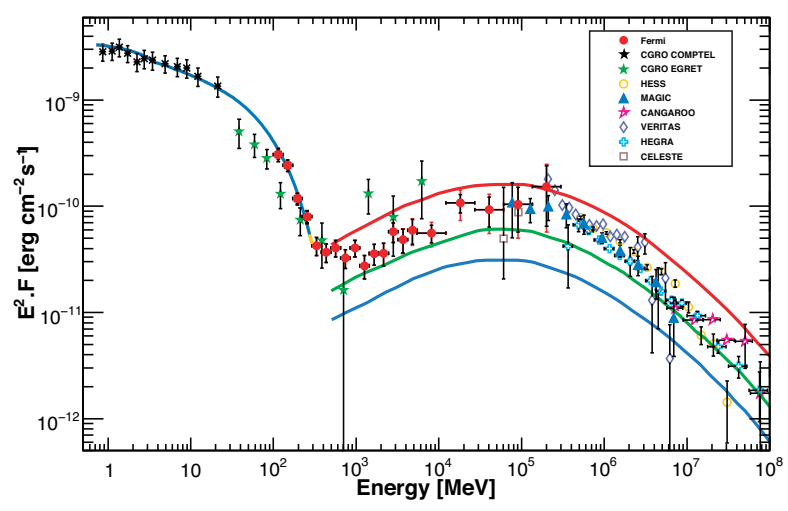

Figure 2: Differential flux from the Crab pulsar as measured from different detectors.

\subsection{Crab Nebula and Pulsar}

The Crab Nebula is a SNR with a pulsar at its center. Crab is the standard candle for VHE astrophysics and as such, an important fraction of the observation time from MAGIC and from the other Cherenkov telescopes is devoted to this object. The VHE source appears point-like and its position is consistent with that of the pulsar.

Recently, thanks to a special trigger setup, MAGIC has detected pulsed emission coming from the Crab pulsar above $25 \mathrm{GeV}$, with a statistical significance of $6.4 \sigma$ [5]. This result has revealed a relatively high energy cutoff, indicating that the emission occurs far out in the magnetosphere, hence excluding the polar-cap scenario as a plausible explanation for the high-energy origin. This is also the first time that a pulsed $\gamma$-ray emission is detected from a ground-based telescope, and opens the possibility of a detailed study of the pulsar's energy cutoff, which will help clarifying the mechanism of high energy radiation in pulsars.

The nonpulsed emission is also very relevant, and it extends up to some $100 \mathrm{TeV}$ (at the limit of the sensitivity of the Cherenkov telescopes). The particular characteristics of MAGIC allow measuring the Crab energy spectrum in the continuum at low energies, overlapping with the Fermi satellite (Fig. 2). This fact can permit cross-calibration [11] among the space-based and groundbased detectors, which will decrease the relative energy uncertainty to some $15 \%$ for the latter.

\section{Highlights of extragalactic observations}

MAGIC is the Cherenkov telescope with the lowest energy threshold (and, as a consequence, it can probe the most distant sources); it contributed to the discovery of 8 Active Galactic Nuclei: 1ES 1218+304 [12] at $z=0.182$, Markarian 180 [13] at $z=0.045$, BL Lacertae [14] at $z=0.069$, 1ES 1011+496 [15] at $z=0.212$, PG 1553+113 [16] at $z>0.09$ (co-discovered with H.E.S.S.), S5 0716+71 [17] at $z=0.31 \pm 0.08$, MAGIC J0223+430 [18] at unknown redshift, possibly coincident with the radio galaxy $3 \mathrm{C} 66 \mathrm{~B}$, and last but not least the farthest one, $3 \mathrm{C} 279$ [19], at the 
unexpectedly large redshift of $z=0.54$. MAGIC has studied most of the AGN in its field of view, often detecting new physics features.

One of the main features of the emissions by AGN is variability. Such a variability can be very rapid, of the order of some 10 seconds or so (the typical size of the central black hole can be 100 to 1000 times larger).

\subsection{Blazars detected during optical outbursts}

Observations triggered by high optical states of potential VHE blazars have proven very successful with the detection of Mkn 180, 1ES 1011+496, 3C 279, and recently S5 0716+71. The observation of 1ES 1011+496 was triggered by an optical outburst in March 2007, resulting in a $6.2 \sigma$ detection [15] of a flux $(1.58 \pm 0.32) \times 10^{-11} \mathrm{~cm}^{-2} \mathrm{~s}^{-1}$ above $200 \mathrm{GeV}$. An indication for a long-term optical-VHE correlation comes from the fact that in spring 2007 the VHE $\gamma$-ray flux was about $40 \%$ higher than in spring 2006, when MAGIC had observed this blazar for the first time without finding a significant signal.

In April 2008, the optical telescope supporting MAGIC, KVA, reported a bright optical state of the blazar S5 0716+71, triggering VHE observations which resulted in a $6.8 \sigma$ detection, corresponding to a flux $\approx 10^{-11} \mathrm{~cm}^{-2} \mathrm{~s}^{-1}$ above $400 \mathrm{GeV}$. The source was also in a high X-ray state.

\subsection{Multiwavelength campaigns}

For a better understanding of the emission mechanisms of AGN, simultaneous multiwavelength (MW) observations are essential. MW observations allow the determination of spectral energy distributions (SEDs) spanning over 15 orders of magnitude in energy. MAGIC recently participated in MW campaigns carried out on the blazars Mkn 421, Mkn 501, PG 1553+113, 1ES

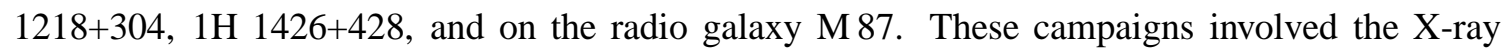
satellites INTEGRAL, Suzaku and Swift, the $\gamma$-ray telescopes H.E.S.S., VERITAS, and MAGIC, and other optical and radio telescopes [6].

Among the main results, a 120-hour observational campaign on the giant radio galaxy M87 in spring 2008 detected two major outbursts of gamma-ray emission, while simultaneous high resolution radio observations of the activity of this radio galaxy were performed. The increase of the radio flux could be located from the innermost core of M87, associated with the immediate vicinity of the central black hole. The combined observation pins down the location of activity during the gamma-ray outbursts and thus the site of the particle accelerator [20].

\section{Impact of the MAGIC results on fundamental physics}

MAGIC has set upper limits on possible signals from the annihilation of Dark Matter particles [21] in channels involving high-energy photons, for the Galactic Center and for the dwarf Spheroidals galaxies Draco and Willman I.

The imaging and the study of the SEDs from Galactic objects by MAGIC and by H.E.S.S. and VERITAS indicate that Galactic sources (PWN in particular) are likely to generate charged cosmic rays up to some $10^{15} \mathrm{eV}$.

We summarize two additional subjects investigated thanks to the study of AGN. 


\subsection{Does the speed of light depend on the photon energy?}

The constancy of the speed of light, $c$, is one of the postulates of the theory of relativity; it has been extensively tested in the recent years against a possible dependence on the photon energy $E$, which is predicted, for example, by theories inspired by Quantum Gravity. At first order, such a dependence is usually parametrized [22] as

$$
c^{\prime}=c\left[1 \pm\left(E / E_{s 1}\right)\right] .
$$

The energy scale $E_{s 1}$ which parametrizes the scale of violation of relativity in the above expression is usually expressed in units of the Planck mass, $M_{P} \simeq 1.22 \times 10^{19} \mathrm{GeV}$.

A favored way to search for such a dispersion relation is to compare the arrival times of photons of different energies arriving on Earth from pulses of distant astrophysical sources. The greatest sensitivities may be expected from sources with short pulses, at large redshifts $z$, of photons observed over a large range of energies: $\gamma$-ray bursts (GRBs) and AGN.

MAGIC [23] has published the results of the analysis of a giant flare of the Mrk 501 blazar, at $z=0.034$. A correlation has been observed (with a probability of $2.5 \%$ of coming from a statistical fluctuation) between the photon energy and the arrival time. The correlation is subluminal, i.e., the minus sign holds in Eq.(4.1) (higher energy photons are delayed) with a coefficient $d t / d E=$ $(0.030 \pm 0.012) \mathrm{s} / \mathrm{GeV}$. Later, the Fermi/LAT detector has registered the most energetic photon, at $E \sim 13.2 \mathrm{GeV}$, from the GRB080916C [24] at $z$ 4.34, $16.5 \mathrm{~s}$ after the primary burst. The delays from the GRB080916C are consistent within errors with the same energy scale [25], although the sources are very different as their distance is.

However, the recent results by Fermi [26] do not fit in the exciting picture outlined above. The most conservative conclusion is that before making any statement we need to understand better the time/energy correlation of the photon emission at the source. Anyway, we proved that the sensitivity of Cherenkov telescopes is such that we could detect effects at the Planck mass scale.

\subsection{Anomalies in the propagation of gamma-rays?}

The horizon of the observable Universe is expected to rapidly shrink in the VHE band as the energy increases, due to the scattering of VHE photons off background photons permeating the Universe, which produces electron-positron pairs [27]. The corresponding cross section $\sigma(\gamma \gamma \rightarrow$ $e^{+} e^{-}$) peaks where the VHE photon energy $E$ and the background photon energy $\varepsilon$ are related by $\varepsilon \simeq(500 \mathrm{GeV} / E) \mathrm{eV}$. Therefore, for observations performed by Cherenkov Telescopes the resulting cosmic opacity is dominated by the interaction with ultraviolet/optical/infrared diffuse background photons, usually called Extragalactic Background Light (EBL), which is produced by galaxies during the whole history of the Universe.

The absorption is usually parametrized as $e^{-\tau(E, z)}$. The mean free path of VHE photons decreases like a power law from the Hubble radius $4.2 \mathrm{Gpc}$ around $100 \mathrm{GeV}$ to $1 \mathrm{Mpc}$ around $100 \mathrm{TeV}$ [28]. Thus, the observed flux is exponentially suppressed both at high energy and at large distances, so that sufficiently far away sources become hardly visible in the VHE range and their observed spectrum should be steeper than the emitted one.

Observations have not detected such a behaviour. Strong evidence comes from the observation of 3C279 (Figure 3, left), which would be expected to be attenuated by a factor $e^{-3}$ at the maxi- 

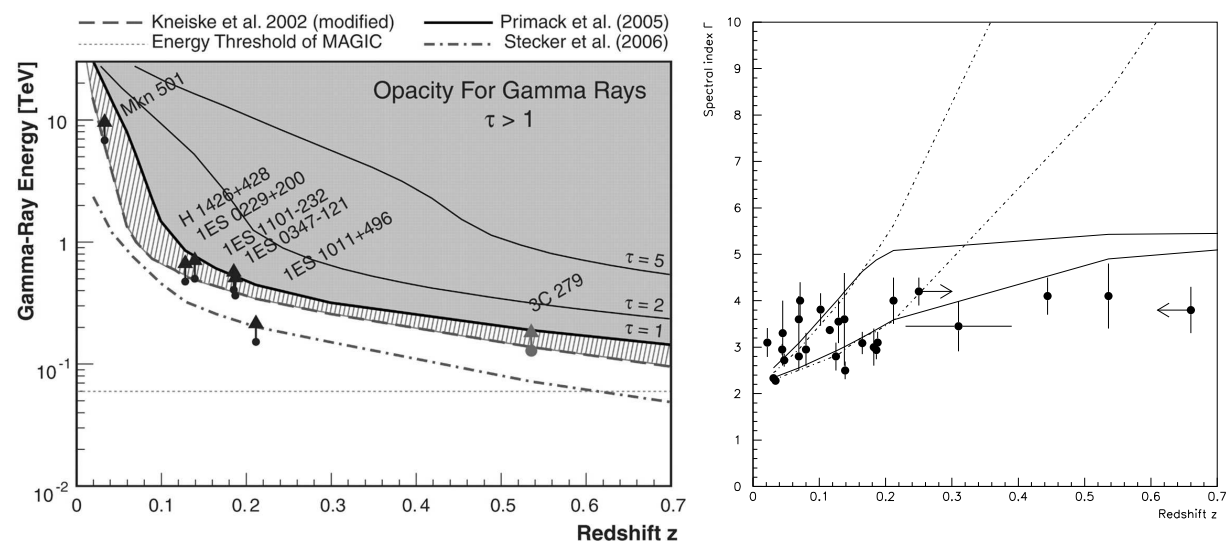

Figure 3: Left: Gamma-ray horizon, from [19], compared to EBL models. The dashed blue line represents an upper EBL limit based on the MAGIC 3C 279 data, obtained on the assumption that the intrinsic spectral index is 1.5. Limits obtained for other sources are shown by black arrows, most of which lie very close to the model. The narrow blue band is the region allowed between this model and a maximum possible transparency (i.e., minimum EBL level), which comes from galaxy counts. The gray area indicates an optical depth $\tau>1$. The curves for $\tau=2$ and $\tau=5$ are also shown. Right: Spectral index $\Gamma_{\text {obs }}$ for blazars observed in the energy band $0.2 \mathrm{TeV}<E_{0}<2 \mathrm{TeV}$ with known redshift. See ref. [29] for references. For a constant spectral index $\Gamma=2.3$ at the emitter, the predictions from the DaRMa model are the band between the solid lines; the standard propagation predicts the band between the dashed lines [30].

mum energies detected of about $400 \mathrm{GeV}$. Another evidence comes from the fact that one does not observe a steepening of the SED of blazars with increasing distances (Figure 3, right).

A possible explanation [30] requires that photons oscillate into a new Axion-Like Parlicle (ALP) and vice-versa in the presence of cosmic magnetic fields (the so-called "DaRMa scenario"); once ALPs are produced close enough to the source, they travel unimpeded throughout the Universe and can convert back to photons before reaching the Earth. Since ALPs do not undergo EBL absorption, the effective photon mean free path is increased. An ALP of mass $\ll 10^{-10} \mathrm{eV}$ could explain the observed results.

\section{Conclusions}

MAGIC at La Palma has discovered many new sources and classes of sources, both galactic and extragalactic. Thanks to its low energy threshold, the lowest among Cherenkov telescopes, it can contribute to many new discoveries, also in the field of fundamental physics. The upgrade to stereoscopy is guaranteeing since end 2009 a further improvement in discovery potential.

\section{Acknowledgements}

I thank Barbara De Lotto, Marcos Lopez, Oriana Mansutti, Massimo Persic, Javi Rico, Marco Roncadelli, Thomas Schweizer and Robert Wagner for providing material for this report, and for useful comments. 


\section{References}

[1] For a review on the Cherenkov technique and experiments see for example A. De Angelis, O. Mansutti and M. Persic, Riv. Nuovo Cim. 31 (2008) 187.

[2] J. Albert et al. (MAGIC Coll.), Astrophys. J. 664 (2007) L87.

[3] J. Albert et al. (MAGIC Coll.), Science 312 (2006) 1771.

[4] J. Albert et al. (MAGIC Coll.), Astrophys. J. 665 (2007) L51.

[5] E. Aliu et al. (MAGIC Coll.), Science 322 (2008) 1221.

[6] For more details on Galactic observations and MW campaigns (Sections 2.1, 2.2, 3.1 and 3.2) see J. Rico and R. Wagner, arXiv:0905.3043 and references therein.

[7] J. Albert et al. (MAGIC Coll.), Astron. Astrophysics 474 (2007) 937.

[8] F.A. Aharonian et al. (HEGRA Coll.), Astron. Astrophysics 112 (2001) 307.

[9] J. Albert et al. (MAGIC Coll.) Astrophys. J. 693 (2009) 303.

[10] J. Albert et al. (MAGIC Coll.), Astrophys. J. 684 (2008) 1351.

[11] D. Bastieri et al., Astropart. Phys. 23 (2005) 572.

[12] J. Albert et al. (MAGIC Coll.), Astrophys. J. 642 (2006) L119.

[13] J. Albert et al. (MAGIC Coll.), Astrophys. J. 648 (2006) L105.

[14] J. Albert et al. (MAGIC Coll.), Astrophys. J. 666 (2007) L17.

[15] J. Albert et al. (MAGIC Coll.), Astrophys. J. 667 (2007) L21.

[16] J. Albert et al. (MAGIC Coll.), Astrophys. J. 654 (2007) L119.

[17] M. Teshima et al. (MAGIC Coll.), ATel 1500 (2008) 1.

[18] E. Aliu et al. (MAGIC Coll.), Astrophys. J. 692 (2009) L29.

[19] J. Albert et al. (MAGIC Coll.), Science 320 (2008) 1752.

[20] V.A. Acciari et al. (H.E.S.S., MAGIC, VERITAS and VLBA Coll.), Science 325 (2009) 444.

[21] S. Lombardi for the MAGIC Collaboration, Contribution to the XXXI ICRC, Lodz 2009.

[22] G. Amelino-Camelia et al., Nature 393 (1998) 763.

[23] J. Albert et al. (MAGIC Coll.), Phys. Lett. B668 (2008) 253.

[24] A.A. Abdo et al. (Fermi Coll.), Science 323 (2009) 1688.

[25] J. Ellis et al., Phys. Lett. B674 (2009) 83.

[26] A.A. Abdo et al. (Fermi Coll.), Nature 462 (2009) 331.

[27] G.G. Fazio and F.W. Stecker, Nature 226135 (1970).

[28] P. Coppi and F. Aharonian, Astrophys. J. 487 (1997) L9.

[29] http://www.mppmu.mpg.de/rwagner/sources/ and references therein.

[30] A. De Angelis, M. Roncadelli and O. Mansutti, Phys. Rev. D76(2007) 121301; A. De Angelis et al., Mon. Not. R. Astron. Soc. 394 (2009) L21 (updated in M. Roncadelli et al., Contribution to SciNeGHE 2009, to appear in the Proceedings). The EBL models used, e.g., in Figure 3, right, are from F. Stecker, M. Malkan and S. Scully, Astrophys. J. 648 (2006) 774. 